Journal of Mathematics and Statistics 5 (1): 42-46, 2009

ISSN 1549-3644

(C) 2009 Science Publications

\title{
Stochastic Behavior of a Two-Unit Warm Standby System with Two Types of Repairmen and Patience Time
}

\author{
${ }^{1}$ G.S. Mokaddis, ${ }^{2}$ M. Salah EL-Sherbeny and ${ }^{3}$ Y. Mahmoud Ayid \\ ${ }^{1}$ Department of Mathematics, Ain shams University, Faculty of Science, Cairo, Egypt \\ ${ }^{2}$ Department of Mathematics, Helwan University, Faculty of Science, Cairo, Egypt \\ ${ }^{3}$ Department of Statistics, Al-Madina Academy, Giza, Egypt
}

\begin{abstract}
Problem statement: In this study we investigated the probabilistic analysis of a two-unit warm standby system with constant failure rate and two types of repairmen and patience time. The first repairman, usually called regular repairman, always remained with the repair facility, with the known fact that he might not be able to do some complex repairs within some tolerable (patience) time. An expert repairman was called for the system if and only if the regular repairman is unable to do the job, within some fixed time or on system failure, whichever occurs first. Approach: Techniques of regenerative point processes had been used to measure the system effectiveness Results: The timedependent availability, steady-state availability, reliability, (MTTF) and profit function were obtained numerically and graphically. Conclusion: The availability and profit decreased with respect to the increase of failure rate.
\end{abstract}

Key words: Availability, busy period, expected number of calls repairman

\section{INTRODUCTION}

Reliability is an important concept at the planning design and operation stages of various complex systems. As long as man has been building things, he has wanted to make them as reliable as possible. In this study we investigate the "probabilistic analysis of a two-unit warm standby system with two types of repairmen and patience time". Although the concept of stochastic behavior of a two-unit cold standby system with two types of repairmen and patience time was discussed $b^{[4]}$, the concept of two type of failure subject to hardware and human error failures was discussed by ${ }^{[1]}$, the concept of probabilistic analysis of two unit warm standby system was discussed by ${ }^{[1,3]}$ and the concept of two-dissimilar unit cold standby redundant system was discussed by ${ }^{[2]}$. This study deals with the reliability and availability analysis of system which has two similar units' one is operative and the other is warm standby. We will introduce the concept of patience time with two types of repairmen for a two unit warm standby system, with different repair rates for regular and expert repairmen. The regular repairman is always available in the repair facility, with the known fact that he might not be able to do some complex repairs within some specified time. The patience time is that for which one can wait while the regular repairman is undergoing repair for the failed unit. The expert repairman is called to the system when a regular repairman is not able to do some complex repairs. The expert repairman leaves the system after completing the repair of the failed unit and the other unit which fails will be attended first by the regular repairman.

\section{MATERIALS AND METHODS}

In this study we analyzed the probabilistic analysis of the system by using of the regenerative processes and have obtained expressions for the various measure of system effectiveness such as the time dependent availability, steady state availability, total fraction of busy period for the regular and expert repairman and total number of visits by the expert repairman per unit time. Using the above measures, profit was calculated. Numerical expression for steady state availability and the profit function were obtained and graphs are also drawn for various parameters involved in the system. We have compared the characteristic, availability and profit with respect to failure rate of the system, to determine when they are improved.

Model description and assumptions: The following assumptions are associated with the model:

Corresponding Author: G.S. Mokaddis, Department of Mathematics, Ain shams University, Faculty of Science, Cairo, Egypt 
- The system consists of two identical units. Initially one unit is operative and the other unit is warm standby

- Failure of the unit is detected and is attended by the regular repairman

- When failure of a unit is detected, repair of failed unit and switching from the standby starts simultaneously. Switchover is perfect and instantaneous

- System is down when both units are fail

- When the expert repairman is called on to do his job, it takes negligible time to reach the system

- The expert repairman leaves the system after completing repair the failed unit. The other unit which fails will be attended to the regular repairman

- After repair the unit work as good as new

- There is a single repair facility attended by two repairman, (regular and expert)

The system can take one of the following states:

$\mathrm{S}_{0}(\mathrm{O}, \mathrm{ST}), \mathrm{S}_{1}\left(\mathrm{U}_{\mathrm{r}}, \mathrm{O}\right), \mathrm{S}_{2}\left(\mathrm{O}, \mathrm{SU}_{\mathrm{r}}\right), \mathrm{S}_{3}\left(\mathrm{U}_{\mathrm{e}}, \mathrm{O}\right), \mathrm{S}_{4}\left(\mathrm{O}, \mathrm{SU}_{\mathrm{e}}\right)$, $\mathrm{S}_{5}\left(\mathrm{U}_{\mathrm{r}}, \mathrm{W}_{\mathrm{r}}\right), \mathrm{S}_{6}\left(\mathrm{~W}_{\mathrm{r}}, \mathrm{SU}_{\mathrm{r}}\right), \mathrm{S}_{7}\left(\mathrm{U}_{\mathrm{e}}, \mathrm{W}_{\mathrm{r}}\right), \mathrm{S}_{8}\left(\mathrm{~W}_{\mathrm{r}}, \mathrm{SU}_{\mathrm{e}}\right)$

Where:

$\mathrm{O}=$ The unit is operative

ST $=$ The unit is standby

$\mathrm{U}_{\mathrm{r}}=$ The operative unit is under regular repairman

$\mathrm{U}_{\mathrm{e}}=$ The operative unit is under expert repairman

$\mathrm{W}_{\mathrm{r}}=$ The unit is waiting for the regular repairman

$\mathrm{SU}_{\mathrm{r}}=$ The stand by unit is under regular repairman

$\mathrm{SU}_{\mathrm{e}}=$ The stand by unit is under expert repairman

\section{RESULTS AND DISCUSSION}

Reliability analysis: The equations determining the reliability of the system can be easily obtained from the following:

$$
\begin{aligned}
& \mathrm{R}_{0}(\mathrm{t})=\mathrm{E}_{1}(\mathrm{t})+\mathrm{E}_{2}(\mathrm{t}) \odot \mathrm{R}_{1}(\mathrm{t})+\mathrm{E}_{3}(\mathrm{t}) \odot \mathrm{R}_{2}(\mathrm{t}), \\
& \mathrm{R}_{1}(\mathrm{t})=\mathrm{E}_{4}(\mathrm{t})+\mathrm{E}_{5}(\mathrm{t}) \odot \mathrm{R}_{0}(\mathrm{t})+\mathrm{E}_{6}(\mathrm{t}) \odot \mathrm{R}_{3}(\mathrm{t}), \\
& \mathrm{R}_{2}(\mathrm{t})=\mathrm{E}_{8}(\mathrm{t})+\mathrm{E}_{9}(\mathrm{t}) \odot \mathrm{R}_{0}(\mathrm{t})+\mathrm{E}_{10}(\mathrm{t}) \odot \mathrm{R}_{4}(\mathrm{t}), \\
& \mathrm{R}_{3}(\mathrm{t})=\mathrm{E}_{12}(\mathrm{t})+\mathrm{E}_{13}(\mathrm{t}) \odot \mathrm{R}_{0}(\mathrm{t}), \\
& \mathrm{R}_{4}(\mathrm{t})=\mathrm{E}_{15}(\mathrm{t})+\mathrm{E}_{16}(\mathrm{t}) \odot \mathrm{R}_{0}(\mathrm{t})
\end{aligned}
$$

By using Laplace transform technique, we can solve for $\mathrm{R}_{0}^{*}(\mathrm{~s})$ and is given by:

$$
\mathrm{R}_{0}^{*}(\mathrm{~s})=\frac{\mathrm{N}(\mathrm{s})}{\mathrm{D}(\mathrm{s})}
$$

Where:

$$
\begin{aligned}
\mathrm{N}(\mathrm{s})= & \mathrm{E}_{1}^{*}(\mathrm{~s})+\mathrm{E}_{3}^{*}(\mathrm{~s})\left(\mathrm{E}_{10}^{*}(\mathrm{~s}) \mathrm{E}_{15}^{*}(\mathrm{~s})+\mathrm{E}_{8}^{*}(\mathrm{~s})\right)+\mathrm{E}_{2}^{*}(\mathrm{~s})\left(\mathrm{E}_{6}^{*}(\mathrm{~s}) \mathrm{E}_{12}^{*}\right. \\
& \left.(\mathrm{s})+\mathrm{E}_{4}^{*}(\mathrm{~s})\right) \\
\mathrm{D}(\mathrm{s})= & 1-\mathrm{E}_{3}^{*}(\mathrm{~s})\left(\mathrm{E}_{10}^{*}(\mathrm{~s}) \mathrm{E}_{16}^{*}(\mathrm{~s})+\mathrm{E}_{9}^{*}(\mathrm{~s})\right)-\mathrm{E}_{2}^{*}(\mathrm{~s})\left(\mathrm{E}_{6}^{*}(\mathrm{~s}) \mathrm{E}_{13}^{*}(\mathrm{~s})\right. \\
& \left.+\mathrm{E}_{5}^{*}(\mathrm{~s})\right)
\end{aligned}
$$

Availability analysis: We have:

$$
\mathrm{A}_{0}(\mathrm{t})=\mathrm{E}_{1}(\mathrm{t})+\mathrm{E}_{2}(\mathrm{t}) \odot \mathrm{A}_{1}(\mathrm{t})+\mathrm{E}_{3}(\mathrm{t}) \odot \mathrm{A}_{2}(\mathrm{t})
$$

The above equation is obtained by considering the two mutually and exhaustive cases:

- No online unit or standby unit fails upon time t

- The online unit fails and hence, the regenerative state 1 occurs

- The standby unit fails and hence, the regenerative state 2 occurs

$$
\begin{aligned}
& \mathrm{A}_{1}(\mathrm{t})=\mathrm{E}_{4}(\mathrm{t})+\mathrm{E}_{5}(\mathrm{t}) \odot \mathrm{A}_{0}(\mathrm{t})+\mathrm{E}_{6}(\mathrm{t}) \odot \mathrm{A}_{3}(\mathrm{t})+\mathrm{E}_{7}(\mathrm{t}) \odot \mathrm{A}_{5}(\mathrm{t}), \\
& \mathrm{A}_{2}(\mathrm{t})=\mathrm{E}_{8}(\mathrm{t})+\mathrm{E}_{9}(\mathrm{t}) \odot \mathrm{A}_{0}(\mathrm{t})+\mathrm{E}_{10}(\mathrm{t}) \odot \mathrm{A}_{4}(\mathrm{t})+\mathrm{E}_{11}(\mathrm{t}) \odot \mathrm{A}_{6}(\mathrm{t}), \\
& \mathrm{A}_{3}(\mathrm{t})=\mathrm{E}_{12}(\mathrm{t})+\mathrm{E}_{13}(\mathrm{t}) \oplus \mathrm{A}_{0}(\mathrm{t})+\mathrm{E}_{14}(\mathrm{t}) \oplus \mathrm{A}_{7}(\mathrm{t}), \\
& \mathrm{A}_{4}(\mathrm{t})=\mathrm{E}_{15}(\mathrm{t})+\mathrm{E}_{16}(\mathrm{t}) \odot \mathrm{A}_{0}(\mathrm{t})+\mathrm{E}_{17}(\mathrm{t}) \odot \mathrm{A}_{8}(\mathrm{t}), \\
& \mathrm{A}_{5}(\mathrm{t})=\mathrm{E}_{18}(\mathrm{t}) \odot \mathrm{A}_{1}(\mathrm{t})+\mathrm{E}_{19}(\mathrm{t}) \odot \mathrm{A}_{7}(\mathrm{t}) \text {, } \\
& A_{6}(t)=E_{20}(t) \odot A_{1}(t)+E_{21}(t) \odot A_{8}(t), \\
& A_{7}(t)=g_{e 1}(t) \odot A_{1}(t), \\
& \mathrm{A}_{8}(\mathrm{t})=\mathrm{g}_{\mathrm{e} 2}(\mathrm{t}) \odot \mathrm{A}_{1}(\mathrm{t})
\end{aligned}
$$

Using Laplace transform technique, we can solve for $\mathrm{A}_{0}^{*}(\mathrm{~s})$ and is given by:

$$
\mathrm{A}_{0}^{*}(\mathrm{~s})=\frac{\mathrm{N}_{1}(\mathrm{~s})}{\mathrm{D}_{1}(\mathrm{~s})}
$$

Where:

$$
\begin{aligned}
\mathrm{N}_{1}(\mathrm{~s})= & A \mathrm{E}_{1}^{*}(\mathrm{~s})+\mathrm{BE}_{2}^{*}(\mathrm{~s})+\mathrm{E}_{3}^{*}(\mathrm{~s})\left[\mathrm{A}\left(\mathrm{E}_{8}^{*}(\mathrm{~s})+\mathrm{E}_{10}^{*}(\mathrm{~s}) \mathrm{E}_{15}^{*}(\mathrm{~s})\right)\right. \\
& +\mathrm{BU}] \\
\mathrm{D}_{1}(\mathrm{~s})= & \mathrm{A}-\mathrm{CE}_{2}^{*}(\mathrm{~s})-\mathrm{E}_{3}^{*}(\mathrm{~s})\left[\mathrm{A}\left(\mathrm{E}_{9}^{*}(\mathrm{~s})+\mathrm{E}_{10}^{*}(\mathrm{~s}) \mathrm{E}_{16}^{*}(\mathrm{~s})\right)+\mathrm{CU}\right]
\end{aligned}
$$

With the steady-state availability of the system given by:

$$
\mathrm{A}_{0}(0)=\lim _{\mathrm{s} \rightarrow 0} \mathrm{~s} \mathrm{~A}_{0}^{*}(\mathrm{~s})=\lim _{\mathrm{t} \rightarrow \infty} \mathrm{A}_{0}(\mathrm{t})
$$

Busy period analysis for regular repairman: Using the usual definition of $\mathrm{B}_{\mathrm{i}}(\mathrm{t})$ for a regular repairman and considering probabilistic arguments, the following recursive relation can be obtained as: 


$$
\begin{aligned}
& \mathrm{B}_{0}(\mathrm{t})=\mathrm{E}_{2}(\mathrm{t}) \odot \mathrm{B}_{1}(\mathrm{t})+\mathrm{E}_{3}(\mathrm{t}) \odot \mathrm{B}_{2}(\mathrm{t}), \\
& \mathrm{B}_{1}(\mathrm{t})=\mathrm{E}_{4}(\mathrm{t})+\mathrm{E}_{5}(\mathrm{t}) \odot \mathrm{B}_{0}(\mathrm{t})+\mathrm{E}_{6}(\mathrm{t}) \odot \mathrm{B}_{3}(\mathrm{t})+\mathrm{E}_{7}(\mathrm{t}) \odot \mathrm{B}_{5}(\mathrm{t}), \\
& \mathrm{B}_{2}(\mathrm{t})=\mathrm{E}_{8}(\mathrm{t})+\mathrm{E}_{9}(\mathrm{t}) \odot \mathrm{B}_{0}(\mathrm{t})+\mathrm{E}_{10}(\mathrm{t}) \odot \mathrm{B}_{4}(\mathrm{t})+\mathrm{E}_{11}(\mathrm{t}) \odot \mathrm{B}_{6}(\mathrm{t}), \\
& \mathrm{B}_{3}(\mathrm{t})=\mathrm{E}_{13}(\mathrm{t}) \odot \mathrm{B}_{0}(\mathrm{t})+\mathrm{E}_{14}(\mathrm{t}) \odot \mathrm{B}_{7}(\mathrm{t}) \text {, } \\
& \mathrm{B}_{4}(\mathrm{t})=\mathrm{E}_{16}(\mathrm{t}) \odot \mathrm{B}_{0}(\mathrm{t})+\mathrm{E}_{17}(\mathrm{t}) \odot \mathrm{B}_{8}(\mathrm{t}), \\
& \mathrm{B}_{5}(\mathrm{t})=\mathrm{E}_{22}(\mathrm{t})+\mathrm{E}_{18}(\mathrm{t}) \odot \mathrm{B}_{1}(\mathrm{t})+\mathrm{E}_{19}(\mathrm{t}) \odot \mathrm{B}_{7}(\mathrm{t}), \\
& \mathrm{B}_{6}(\mathrm{t})=\mathrm{E}_{23}(\mathrm{t})+\mathrm{E}_{20}(\mathrm{t}) \odot \mathrm{B}_{1}(\mathrm{t})+\mathrm{E}_{21}(\mathrm{t}) \odot \mathrm{B}_{8}(\mathrm{t}), \\
& \mathrm{B}_{7}(\mathrm{t})=\mathrm{g}_{\mathrm{e} 1}(\mathrm{t}) \odot \mathrm{B}_{1}(\mathrm{t}) \text {, } \\
& \mathrm{B}_{8}(\mathrm{t})=\mathrm{g}_{\mathrm{e} 2}(\mathrm{t}) \odot \mathrm{B}_{1}(\mathrm{t})
\end{aligned}
$$

Using Laplace transform technique, we can solve for $\mathrm{B}_{0}^{*}(\mathrm{~s})$ we get:

$$
\begin{aligned}
\mathrm{BE}_{2}^{*}(\mathrm{~s})+\mathrm{E}_{3}^{*}(\mathrm{~s})\left[\mathrm{A}\left(\mathrm{E}_{8}^{*}(\mathrm{~s})+\mathrm{E}_{11}^{*}(\mathrm{~s}) \mathrm{E}_{23}^{*}(\mathrm{~s})\right)\right. \\
\mathrm{B}_{0}^{*}(\mathrm{~s})=\frac{\left.+\mathrm{U}\left(\mathrm{E}_{4}^{*}(\mathrm{~s})+\mathrm{E}_{7}^{*}(\mathrm{~s}) \mathrm{E}_{22}^{*}(\mathrm{~s})\right)\right]}{\mathrm{D}_{1}(\mathrm{~s})}
\end{aligned}
$$

The steady-state of idle time of the regular repairman is given by:

$$
B_{0}(0)=\lim _{s \rightarrow 0} s B_{0}^{*}(s)=\lim _{t \rightarrow \infty} B_{0}(t)
$$

Busy period analysis for expert repairman: Using the usual definition of $\mathrm{T}_{\mathrm{i}}(\mathrm{t})$ for an expert repairman and considering probabilistic arguments, the following recursive relation can be obtained as:

$$
\begin{aligned}
& \mathrm{T}_{0}(\mathrm{t})=\mathrm{E}_{2}(\mathrm{t}) \odot \mathrm{T}_{1}(\mathrm{t})+\mathrm{E}_{3}(\mathrm{t}) \odot \mathrm{T}_{2}(\mathrm{t}), \\
& \mathrm{T}_{1}(\mathrm{t})=\mathrm{E}_{5}(\mathrm{t}) \odot \mathrm{T}_{0}(\mathrm{t})+\mathrm{E}_{6}(\mathrm{t}) \odot \mathrm{T}_{3}(\mathrm{t})+\mathrm{E}_{7}(\mathrm{t}) \odot \mathrm{T}_{5}(\mathrm{t}), \\
& \mathrm{T}_{2}(\mathrm{t})=\mathrm{E}_{9}(\mathrm{t}) \odot \mathrm{T}_{0}(\mathrm{t})+\mathrm{E}_{10}(\mathrm{t}) \odot \mathrm{T}_{4}(\mathrm{t})+\mathrm{E}_{11}(\mathrm{t}) \odot \mathrm{T}_{6}(\mathrm{t}), \\
& \mathrm{T}_{3}(\mathrm{t})=\mathrm{E}_{12}(\mathrm{t})+\mathrm{E}_{13}(\mathrm{t}) \odot \mathrm{T}_{0}(\mathrm{t})+\mathrm{E}_{14}(\mathrm{t}) \odot \mathrm{T}_{7}(\mathrm{t}), \\
& \mathrm{T}_{4}(\mathrm{t})=\mathrm{E}_{15}(\mathrm{t})+\mathrm{E}_{16}(\mathrm{t}) \odot \mathrm{T}_{0}(\mathrm{t})+\mathrm{E}_{17}(\mathrm{t}) \odot \mathrm{T}_{8}(\mathrm{t}), \\
& \mathrm{T}_{5}(\mathrm{t})=\mathrm{E}_{18}(\mathrm{t}) \odot \mathrm{T}_{1}(\mathrm{t})+\mathrm{E}_{19}(\mathrm{t}) \odot \mathrm{T}_{7}(\mathrm{t}), \\
& \mathrm{T}_{6}(\mathrm{t})=\mathrm{E}_{20}(\mathrm{t}) \odot \mathrm{T}_{1}(\mathrm{t})+\mathrm{E}_{21}(\mathrm{t}) \odot \mathrm{T}_{8}(\mathrm{t}), \\
& \mathrm{T}_{7}(\mathrm{t})=\overline{\mathrm{G}}_{\mathrm{e} 1}(\mathrm{t})+\mathrm{g}_{\mathrm{e} 1}(\mathrm{t}) \odot \mathrm{T}_{1}(\mathrm{t}) \\
& \mathrm{T}_{8}(\mathrm{t})=\overline{\mathrm{G}}_{\mathrm{e} 2}(\mathrm{t})+\mathrm{g}_{\mathrm{e} 2}(\mathrm{t}) \odot \mathrm{T}_{1}(\mathrm{t})
\end{aligned}
$$

Using Laplace transform technique, we can solve for $\mathrm{T}_{0}^{*}(\mathrm{~s})$ we get:

$$
\begin{array}{r}
\mathrm{LE}_{2}^{*}(\mathrm{~s})+\mathrm{E}_{3}^{*}(\mathrm{~s})\left(\mathrm { A } \left(\mathrm{E}_{10}^{*}(\mathrm{~s})\left(\mathrm{E}_{15}^{*}(\mathrm{~s})+\mathrm{E}_{17}^{*}(\mathrm{~s}) \mathrm{G}_{\mathrm{e} 2}^{*}(\mathrm{~s})\right)\right.\right. \\
\mathrm{T}_{0}^{*}(\mathrm{~s})=\frac{\left.\left.+\mathrm{E}_{11}^{*}(\mathrm{~s}) \mathrm{E}_{21}^{*}(\mathrm{~s}) \mathrm{G}_{\mathrm{e} 2}^{*}(\mathrm{~s})\right)+\mathrm{LU}\right)}{\mathrm{D}_{1}(\mathrm{~s})}
\end{array}
$$

The steady-state of idle time of the expert repairman is given by:

$$
\mathrm{T}_{0}(0)=\lim _{\mathrm{s} \rightarrow 0} \mathrm{~s} \mathrm{~T}_{0}^{*}(\mathrm{~s})=\lim _{\mathrm{t} \rightarrow \infty} \mathrm{T}_{0}(\mathrm{t})
$$

Expected number of calls for the expert repairman: Using the usual definition the following recursive relation for $\mathrm{V}_{\mathrm{i}}(\mathrm{t})$ can be obtained as:

$$
\begin{aligned}
& \mathrm{V}_{0}(\mathrm{t})=\mathrm{E}_{2}(\mathrm{t}) \odot \mathrm{V}_{1}(\mathrm{t})+\mathrm{E}_{3}(\mathrm{t}) \odot \mathrm{V}_{2}(\mathrm{t}), \\
& \mathrm{V}_{1}(\mathrm{t})=\mathrm{E}_{5}(\mathrm{t}) \odot \mathrm{V}_{0}(\mathrm{t})+\mathrm{E}_{6}(\mathrm{t}) \odot\left(1+\mathrm{V}_{3}(\mathrm{t})\right)+\mathrm{E}_{7}(\mathrm{t}) \odot \mathrm{V}_{5}(\mathrm{t}), \\
& \mathrm{V}_{2}(\mathrm{t})=\mathrm{E}_{9}(\mathrm{t}) \odot \mathrm{V}_{0}(\mathrm{t})+\mathrm{E}_{10}(\mathrm{t}) \odot\left(1+\mathrm{V}_{4}(\mathrm{t})\right)+\mathrm{E}_{11}(\mathrm{t}) \odot \mathrm{V}_{6}(\mathrm{t}), \\
& \mathrm{V}_{3}(\mathrm{t})=\mathrm{E}_{13}(\mathrm{t}) \odot \mathrm{V}_{0}(\mathrm{t})+\mathrm{E}_{14}(\mathrm{t}) \odot \mathrm{V}_{7}(\mathrm{t}), \\
& \mathrm{V}_{4}(\mathrm{t})=\mathrm{E}_{16}(\mathrm{t}) \odot \mathrm{V}_{0}(\mathrm{t})+\mathrm{E}_{17}(\mathrm{t}) \odot \mathrm{V}_{8}(\mathrm{t}), \\
& \mathrm{V}_{5}(\mathrm{t})=\mathrm{E}_{18}(\mathrm{t}) \odot \mathrm{V}_{1}(\mathrm{t})+\mathrm{E}_{19}(\mathrm{t}) \odot\left(1+\mathrm{V}_{7}(\mathrm{t})\right) \\
& \mathrm{V}_{6}(\mathrm{t})=\mathrm{E}_{20}(\mathrm{t}) \odot \mathrm{V}_{1}(\mathrm{t})+\mathrm{E}_{21}(\mathrm{t}) \odot\left(1+\mathrm{V}_{8}(\mathrm{t})\right) \\
& \mathrm{V}_{7}(\mathrm{t})=\mathrm{g}_{\mathrm{e} 1}(\mathrm{t}) \odot \mathrm{V}_{1}(\mathrm{t}), \\
& \mathrm{V}_{8}(\mathrm{t})=\mathrm{g}_{\mathrm{e} 2}(\mathrm{t}) \odot \mathrm{V}_{1}(\mathrm{t})
\end{aligned}
$$

Using Laplace transform technique, we can solve for $\mathrm{V}_{0}^{*}(\mathrm{~s})$ we get:

$$
\mathrm{V}_{0}^{*}(\mathrm{~s})=\frac{\mathrm{KE}_{2}^{*}(\mathrm{~s})+\mathrm{E}_{3}^{*}(\mathrm{~s})\left[\mathrm{A}\left(\mathrm{E}_{10}^{*}(\mathrm{~s})+\mathrm{E}_{11}^{*}(\mathrm{~s}) \mathrm{E}_{21}^{*}(\mathrm{~s})\right)+\mathrm{KU}\right]}{\mathrm{D}_{1}(\mathrm{~s})}
$$

The steady-state of the expected number of calls for repairman per unit time is given by:

$$
\mathrm{V}_{0}(0)=\lim _{\mathrm{s} \rightarrow 0} \mathrm{~s}^{2} \mathrm{~V}_{0}^{*}(\mathrm{~s})=\lim _{\mathrm{t} \rightarrow \infty} \frac{\mathrm{V}_{0}(\mathrm{t})}{\mathrm{t}}
$$

Profit analysis: Now the expected profit $\mathrm{P}(\mathrm{t})$ per unit time incurred to the system is given by:

$$
\mathrm{P}(\mathrm{t})=\mathrm{C}_{1} \mu_{\text {up }}(\mathrm{t})-\mathrm{C}_{2} \mu_{\mathrm{r}}(\mathrm{t})-\mathrm{C}_{3} \mu_{\mathrm{e}}(\mathrm{t})-\mathrm{C}_{4} \mathrm{~V}_{0}(\mathrm{t})
$$

Where:

$\mathrm{C}_{1}=$ The revenue per unit up-time of the system

$\mathrm{C}_{2}=$ The cost per unit time for which the regular repairman is busy

$\mathrm{C}_{3}=$ The cost per unit time for which the expert repairman is busy

$\mathrm{C}_{4}=$ The cost per call for the expert repairman

Where:

$$
\begin{aligned}
& \mathrm{A}=1-\mathrm{E}_{7}^{*}(\mathrm{~s})\left(\mathrm{E}_{18}^{*}(\mathrm{~s})+\mathrm{E}_{19}^{*}(\mathrm{~s}) \mathrm{g}_{\mathrm{e} 1}^{*}(\mathrm{~s})\right)-\mathrm{E}_{6}^{*}(\mathrm{~s}) \mathrm{E}_{14}^{*}(\mathrm{~s}) \mathrm{g}_{\mathrm{e} 1}^{*}(\mathrm{~s}) \\
& \mathrm{B}=\mathrm{E}_{4}^{*}(\mathrm{~s})+\mathrm{E}_{6}^{*}(\mathrm{~s}) \mathrm{E}_{12}^{*}(\mathrm{~s}) \\
& \mathrm{C}=\mathrm{E}_{5}^{*}(\mathrm{~s})+\mathrm{E}_{6}^{*}(\mathrm{~s}) \mathrm{E}_{13}^{*}(\mathrm{~s}) \\
& \mathrm{U}=\mathrm{E}_{11}^{*}(\mathrm{~s})\left(\mathrm{E}_{20}^{*}(\mathrm{~s})+\mathrm{E}_{21}^{*}(\mathrm{~s}) \mathrm{g}_{\mathrm{e} 2}^{*}(\mathrm{~s})\right)+\mathrm{E}_{10}^{*}(\mathrm{~s}) \mathrm{E}_{17}^{*}(\mathrm{~s}) \mathrm{g}_{\mathrm{e} 2}^{*}(\mathrm{~s}) \\
& \mathrm{L}=\mathrm{E}_{6}^{*}(\mathrm{~s}) \mathrm{E}_{12}^{*}(\mathrm{~s})+\mathrm{G}_{\mathrm{e} 1}^{*}(\mathrm{~s})\left(\mathrm{E}_{6}^{*}(\mathrm{~s}) \mathrm{E}_{14}^{*}(\mathrm{~s})+\mathrm{E}_{7}^{*}(\mathrm{~s}) \mathrm{E}_{19}^{*}(\mathrm{~s})\right) \\
& \mathrm{K}=\mathrm{E}_{6}^{*}(\mathrm{~s})+\mathrm{E}_{7}^{*}(\mathrm{~s}) \mathrm{E}_{19}^{*}(\mathrm{~s})
\end{aligned}
$$

Numerical analysis: the results obtained for the above model are illustrated with numerical example, we assume that $\mathrm{f}_{\mathrm{i}}(\mathrm{t})=\lambda_{\mathrm{i}} \mathrm{e}^{-\lambda_{\mathrm{i}} \mathrm{t}}, \mathrm{g}_{\mathrm{ji}}(\mathrm{t})=\mu_{\mathrm{ji}} \mathrm{e}^{-\mu_{\mathrm{ji}} \mathrm{t}}, \mathrm{h}_{\mathrm{i}}(\mathrm{t})=\mathrm{a}_{\mathrm{i}} \mathrm{e}^{-\mathrm{a}_{\mathrm{i}} \mathrm{t}}$, where $i=1,2 j=r, e$.

Reliability analysis: The mean time to system failure is give by: 


$$
\mathrm{MTTF}=\operatorname{Lim}_{S \rightarrow 0} R_{0}^{*}(S)=\frac{\mathrm{N}(0)}{\mathrm{D}(0)}
$$

Where:

$$
\begin{aligned}
\mathrm{N}(0)= & \left(\lambda_{1}+\mu_{\mathrm{r} 1}+\mathrm{a}_{1}\right)\left(\lambda_{1}+\mu_{\mathrm{r} 2}+\mathrm{a}_{2}\right)\left[\left(\lambda_{1}+\mu_{\mathrm{r} 2}+\mathrm{a}_{2}\right)\left(\lambda_{1}+\mu_{\mathrm{e} 1}\right)+\lambda_{1}\right. \\
& \left.\left(\lambda_{1}+\mu_{\mathrm{e} 2}\right)+\lambda_{2}\left(\lambda_{1}+\mu_{\mathrm{e} 1}\right)\right] \\
\mathrm{D}(0)= & \left(\lambda_{1}+\lambda_{2}\right)\left(\lambda_{1}+\mu_{\mathrm{e}}\right)\left(\lambda_{1}+\mu_{\mathrm{e} 2}\right)\left(\lambda_{1}+\mu_{\mathrm{r} 1}+\mathrm{a}_{1}\right)\left(\lambda_{1}+\mu_{\mathrm{r} 2}+\right. \\
& \left.\mathrm{a}_{2}\right)-\lambda_{1}\left(\lambda_{1}+\mu_{\mathrm{e} 2}\right)\left(\lambda_{1}+\mu_{\mathrm{r} 2}+\mathrm{a}_{2}\right)\left(\mathrm{a}_{1} \mu_{\mathrm{e}}+\mu_{\mathrm{r} 1}\left(\lambda_{1}+\mu_{\mathrm{e} 1}\right)\right)-\lambda_{2} \\
& \left(\lambda_{1}+\mu_{\mathrm{e} 1}\right)\left(\lambda_{1}+\mu_{\mathrm{r} 1}+\mathrm{a}_{1}\right)\left(\mathrm{a}_{2} \mu_{\mathrm{e} 2}+\mu_{\mathrm{r} 2}\left(\lambda_{1}+\mu_{\mathrm{e} 2}\right)\right)
\end{aligned}
$$

Availability analysis: The time dependent availability of the system is given by:

$$
\mathrm{A}_{0}(\mathrm{t})=\frac{\Psi_{1}}{\prod_{\mathrm{i}=1}^{8} \mathrm{~S}_{\mathrm{i}}}+\sum_{\mathrm{i}=1}^{8} \frac{\Psi_{2} \mathrm{e}_{\mathrm{i}} \mathrm{t}}{\prod_{\substack{\mathrm{r} \neq=\mathrm{i} \\ \mathrm{r}=\mathrm{i}}}^{8} \mathrm{~S}_{\mathrm{i}}\left(\mathrm{S}_{\mathrm{i}}-\mathrm{S}_{\mathrm{r}}\right)}
$$

Where:

$$
\begin{aligned}
\Psi_{1}= & \mu_{\mathrm{e} 2} \mu_{\mathrm{e} 1}\left(\mathrm{a}_{2}+\mu_{\mathrm{r} 2}\right)\left(\mathrm{a}_{1}+\mu_{\mathrm{r} 1}\right)\left[\left(\left(\lambda_{1}+\mu_{\mathrm{e} 1}\right)\left(\mathrm{a}_{1}+\mu_{\mathrm{r} 1}\right)-\mathrm{a}_{1} \lambda_{1}\right)\right. \\
& \left(\left(\lambda_{1}+\mu_{\mathrm{e} 2}\right)\left(\lambda_{1}+\lambda_{2}+\mathrm{a}_{2}+\mu_{\mathrm{r}}\right)+\mathrm{a}_{2} \lambda_{2}\right)+\lambda_{1}\left(\lambda_{1}+\mathrm{a}_{1}+\mu_{\mathrm{e} 1}\right) \\
& \left.\left(\left(\lambda_{1}+\mathrm{a}_{2}+\mu_{\mathrm{r} 2}\right)\left(\lambda_{1}+\mu_{\mathrm{e} 2}\right)+\lambda_{2}\left(\lambda_{1}+\mathrm{a}_{2}+\mu_{\mathrm{e} 2}\right)\right)\right] \\
\Psi_{2}= & \left(\left(\mathrm{S}_{\mathrm{i}}+\mu_{\mathrm{e} 1}\right)\left(\mathrm{S}_{\mathrm{i}}+\lambda_{1}+\mu_{\mathrm{e} 1}\right)\left(\mathrm{S}_{\mathrm{i}}+\mathrm{a}_{1}+\mu_{\mathrm{r} 1}\right)\left(\mathrm{S}_{\mathrm{i}}+\lambda_{1}+\mathrm{a}_{1}+\mu_{\mathrm{r} 1}\right)-\right. \\
& \lambda_{1} \mathrm{a}_{1} \mu_{\mathrm{e} 1}\left(2 \mathrm{~S}_{\mathrm{i}}+\lambda_{1}+\mathrm{a}_{1}+\mu_{\mathrm{r} 1}+\mu_{\mathrm{e} 1}\right)-\lambda_{1} \mu_{\mathrm{r} 1}\left(\mathrm{~S}_{\mathrm{i}}+\mu_{\mathrm{e} 1}\right) \\
& \left.\left(\mathrm{S}_{\mathrm{i}}+\lambda_{1}+\mu_{\mathrm{e} 1}\right)\right)\left(( \mathrm { S } _ { \mathrm { i } } + \mathrm { a } _ { 2 } + \mu _ { \mathrm { r } } ) ( \mathrm { S } _ { \mathrm { i } } + \mu _ { \mathrm { e } } ) \left(\left(\mathrm{S}_{\mathrm{i}}+\lambda_{1}+\mu_{\mathrm{e} 2}\right)\right.\right. \\
& \left.\left.\left(\mathrm{S}_{\mathrm{i}}+\lambda_{1}+\mathrm{a}_{2}+\mu_{\mathrm{r} 2}\right)+\lambda_{2}\left(\mathrm{~S}_{\mathrm{S}}+\lambda_{1}+\mathrm{a}_{2}+\mu_{\mathrm{e}}\right)\right)\right)+\lambda_{1}\left(\mathrm{~S}_{\mathrm{i}}+\lambda_{1}+\right. \\
& \left.\mathrm{a}_{1}+\mu_{\mathrm{e} 1}\right)\left(\mathrm{S}_{\mathrm{i}}+\mathrm{a}_{1}+\mu_{\mathrm{r} 1}\right)\left(\left(\mathrm{S}_{\mathrm{i}}+\lambda_{1}+\mu_{\mathrm{e} 2}\right)\left(\mathrm{S}_{\mathrm{i}}+\mathrm{a}_{2}+\mu_{\mathrm{r} 2}\right)\right. \\
& \left(\mathrm{S}_{\mathrm{i}}+\lambda_{1}+\mathrm{a}_{2}+\mu_{\mathrm{r} 2}\right)\left(\mathrm{S}_{\mathrm{i}}+\mu_{\mathrm{e} 2}\right)+\lambda_{2}\left(\mathrm{~S}_{\mathrm{i}}+\lambda_{1}+\mu_{\mathrm{e} 2}\right)\left(\mu_{\mathrm{r} 2}\right. \\
& \left.\left(\mathrm{S}_{\mathrm{i}}+\mu_{\mathrm{e} 2}\right)+\mathrm{a}_{2} \mu_{\mathrm{e} 2}\right)+\mathrm{a}_{2} \mu_{\mathrm{e} 2}\left(\mathrm{~S}_{\mathrm{i}}+\mathrm{a}_{2}+\mu_{\mathrm{r} 2}\right)
\end{aligned}
$$

where, $\mathrm{S}_{\mathrm{i}}$ are roots of the polynomial $\mathrm{D}_{\mathrm{i}}(\mathrm{s})$ given in Eq. 4.

Busy period analysis for regular repairman: The time dependent busy period for the regular repairman of the system is given by:

$$
\mathrm{B}_{0}(\mathrm{t})=\frac{\mathrm{Q}_{1}}{\prod_{\mathrm{i}=1}^{8} \mathrm{~S}_{1}}+\sum_{\mathrm{i}=1}^{8} \frac{\mathrm{Q}_{2} \mathrm{e}_{\mathrm{i}} \mathrm{\textrm {S } _ { \mathrm { t } }}}{\prod_{\substack{\mathrm{r} \neq 1 \\ \mathrm{r} \neq \mathrm{i}}}^{8} \mathrm{~S}_{\mathrm{i}}\left(\mathrm{S}_{\mathrm{i}}-\mathrm{S}_{\mathrm{r}}\right)}
$$

Where:

$$
\begin{aligned}
\mathrm{Q}_{1}= & \mu_{\mathrm{e} 2} \mu_{\mathrm{e} 1}\left(\mathrm{a}_{1}+\mu_{\mathrm{r} 1}\right)\left(\lambda_{1}+\mu_{\mathrm{e} 2}\right)\left(\lambda_{1}+\mathrm{a}_{2}+\mu_{\mathrm{r} 2}\right)\left(\lambda _ { 2 } \left(\mathrm{a}_{1} \mu_{\mathrm{e} 1}+\mu_{\mathrm{r} 1}\right.\right. \\
& \left(\lambda_{1}+\mu_{\mathrm{e} 1}\right)+\left(\lambda_{1}\left(\lambda_{1}+\mathrm{a}_{1}+\mu_{\mathrm{e} 1}\right)\left(\mathrm{a}_{2}+\mu_{\mathrm{r} 2}\right) \lambda_{1}\left(\lambda_{1}+\mathrm{a}_{1}+\mu_{\mathrm{e} 1}\right)\right. \\
& \left(\mathrm{a}_{2}+\mu_{\mathrm{r} 2}\right) \lambda_{1} \lambda_{2}\left(\lambda_{1}+\mu_{\mathrm{e} 1}\right)\left(\mathrm{a}_{2}+\mu_{\mathrm{r} 2}\right)\left(\lambda_{1}+\mathrm{a}_{2}+\mu_{\mathrm{e} 2}\right)+\lambda_{2} \\
& \left(\lambda_{1}+\mathrm{a}_{1}+\mu_{\mathrm{r} 1}\right) \\
\mathrm{Q}_{2}= & \left(\mathrm{Si}+\mu_{\mathrm{e} 2}\right)\left(\mathrm{S}_{\mathrm{i}}+\lambda_{1}+\mu_{\mathrm{e} 2}\right)\left(\lambda_{1}\left(\mathrm{~S}_{\mathrm{i}}+\mathrm{a}_{1}+\lambda_{1}+\mu_{\mathrm{e}}\right)\left(\mathrm{S}_{\mathrm{i}}+\mu_{\mathrm{e} 1}\right)\right. \\
& \left(\mathrm{S}_{\mathrm{i}}+\mathrm{e}_{1}+\mu_{\mathrm{r} 1}\right)\left(\mathrm{S}_{\mathrm{i}}+\mathrm{a}_{2}+\mu_{\mathrm{r} 2}\right)\left(\mathrm{S}_{\mathrm{i}}+\lambda_{1}+\mathrm{a}_{2}+\mu_{\mathrm{r} 2}\right)+\lambda_{2} \mathrm{X} \\
& \left.\left(\mathrm{S}_{\mathrm{i}}+\mathrm{a}_{2}+\mu_{\mathrm{r} 2}\right)\right)+\lambda_{1} \lambda_{2} \mathrm{X}
\end{aligned}
$$

Busy period analysis for expert repairman: The time dependent busy period for the expert repairman of the system is given by:

$$
\mathrm{T}_{0}(\mathrm{t})=\frac{\mathrm{W}_{1}}{\prod_{\mathrm{i}=1}^{8} \mathrm{~S}_{\mathrm{i}}}+\sum_{\mathrm{i}=1}^{8} \frac{\mathrm{W}_{2} \mathrm{e}^{\mathrm{S}_{\mathrm{i}} \mathrm{t}}}{\prod_{\substack{\mathrm{r}=1 \\ \mathrm{r} \neq \mathrm{i}}}^{8} \mathrm{~S}_{(}\left(\mathrm{S}_{\mathrm{i}}-\mathrm{S}_{\mathrm{r}}\right)}
$$

Where:

$$
\begin{aligned}
\mathrm{W}_{1}= & \left(\mathrm{a}_{2}+\mu_{\mathrm{r} 2}\right)\left(\lambda _ { 2 } \mathrm { a } _ { 2 } \mu _ { \mathrm { e } 1 } ( \mathrm { a } _ { 1 } + \mu _ { \mathrm { r } 1 } ) ( \lambda _ { 1 } + \mu _ { \mathrm { e } 2 } ) \left(\mathrm{a}_{1} \mu_{\mathrm{e} 1}+\mu_{\mathrm{r} 1}\right.\right. \\
& \left.\left(\lambda_{1}+\mu_{\mathrm{e} 1}\right)\right)+\lambda_{1} \mathrm{a}_{1} \mu_{\mathrm{e} 2}\left(\lambda_{1}+\mathrm{a}_{2}+\mu_{\mathrm{r} 1}\right)\left(\lambda_{1}+\lambda_{2}+\mu_{\mathrm{e} 2}\right)\left(\mu_{\mathrm{e} 1}\right. \\
& \left.\left.\left(\mathrm{a}_{1}+\mu_{\mathrm{r} 1}\right)+\left(\lambda_{1}+\mathrm{a}_{1}+\mu_{\mathrm{r} 1}+\mu_{\mathrm{e} 1}\right)\right)\right) \\
\mathrm{W}_{2}= & \left(\mathrm { a } _ { 1 } \lambda _ { 1 } \mathrm { Y } \left(( \mathrm { S } _ { \mathrm { i } } + \mu _ { \mathrm { e } 2 } ) ( \mathrm { S } _ { \mathrm { S } } + \lambda _ { 1 } + \mu _ { \mathrm { e } 2 } ) ( \mathrm { S } _ { \mathrm { i } } + \mathrm { a } _ { 2 } + \mu _ { \mathrm { r } 2 } ) \left(\mathrm{S}_{\mathrm{i}}+\lambda_{1}+\right.\right.\right. \\
& \left.\mathrm{a}_{2}+\mu_{\mathrm{r} 2}\right)+\lambda_{2}\left(\mathrm{a}_{2} \mu_{\mathrm{e} 2}\left(\mathrm{~S}_{\mathrm{i}}+\mathrm{a}_{2}+\mu_{\mathrm{r} 2}\right)+\left(\mathrm{S}_{\mathrm{i}}+\lambda_{1}+\mu_{\mathrm{e} 2}\right)\right. \\
& \left.\left(\mathrm{a}_{2} \mu_{\mathrm{e} 2}+\mu_{\mathrm{r} 2}\left(\mathrm{~S}_{\mathrm{i}}+\mu_{\mathrm{e}}\right)\right)\right)+\mathrm{a}_{2} \lambda_{2} \mathrm{X}\left(\mathrm{S}_{\mathrm{i}}+\mathrm{a}_{2}+\mu_{\mathrm{r} 2}\right) \\
& \left.\left(\mathrm{S}_{\mathrm{i}}+\lambda_{1}+\mu_{\mathrm{e} 2}\right)\right)
\end{aligned}
$$

Expected number of calls for the expert repairman: The number of visits by the expert repairman of the system is given by:

$$
\mathrm{V}_{0}(\mathrm{t})=\frac{\mathrm{Z}_{1}}{\prod_{\mathrm{i}=1}^{8} \mathrm{~S}_{\mathrm{i}}}+\sum_{\mathrm{i}=1}^{8} \frac{\mathrm{Z}_{2} \mathrm{e}^{\mathrm{S}_{\mathrm{i}} \mathrm{t}}}{\prod_{\substack{\mathrm{r} \neq 1 \\ \mathrm{r} \neq \mathrm{i}}}^{8} \mathrm{~S}_{\mathrm{i}}\left(\mathrm{S}_{\mathrm{i}}-\mathrm{S}_{\mathrm{r}}\right)}
$$

$$
\begin{aligned}
\mathrm{Z}_{1}= & \mu_{\mathrm{e} 1} \mu_{\mathrm{e} 2}\left(\mathrm{a}_{1} \lambda_{1}\left(\lambda_{1}+\mu_{\mathrm{e} 1}\right)\left(\lambda_{1}+\mu_{\mathrm{e} 2}\right)\left(\mathrm{a}_{2}+\mu_{\mathrm{r} 2}\right)\left(\lambda_{1}+\mathrm{a}_{2}+\mu_{\mathrm{r} 2}\right)\right. \\
& \left(\lambda_{1}+\mathrm{a}_{1}+\mu_{\mathrm{r} 1}\right)+\lambda_{2}\left(\mathrm{a}_{2}\left(\mathrm{a}_{1}+\mu_{\mathrm{r} 1}\right)\left(\lambda_{1}+\mu_{\mathrm{e}}\right)\left(\lambda_{1}+\mathrm{a}_{2}+\mu_{\mathrm{r} 2}\right)\right. \\
& \left(\mathrm{a}_{1} \mu_{\mathrm{r} 2}+\mu_{\mathrm{r} 1}\left(\lambda_{1}+\mu_{\mathrm{e} 1}\right)\right)+\mathrm{a}_{1} \lambda_{1}\left(\mathrm{a}_{2}+\mu_{\mathrm{r} 2}\right)\left(\lambda_{1}+\mu_{\mathrm{e} 1}\right) \\
& \left.\left.\left(\lambda_{1}+\mathrm{a}_{1}+\mu_{\mathrm{r} 1}\right)\left(\lambda_{1}+\mathrm{a}_{2}+\mu_{\mathrm{e} 2}\right)\right)\right) \\
\mathrm{Z}_{2}= & \mathrm{a}_{1} \lambda_{1}\left(\mathrm{~S}_{\mathrm{i}}+\mu_{\mathrm{e} 1}\right)\left(\mathrm{S}_{\mathrm{i}}+\mu_{\mathrm{e}}\right)\left(\mathrm{S}_{\mathrm{i}}+\lambda_{1}+\mu_{\mathrm{e} 1}\right)\left(\mathrm{S}_{\mathrm{i}}+\lambda_{1}+\mu_{\mathrm{e} 2}\right) \\
& \left(\mathrm{S}_{\mathrm{i}}+\mathrm{a}_{2}+\mu_{\mathrm{r} 2}\right)\left(\mathrm{S}_{\mathrm{i}}+\lambda_{1}+\mathrm{a}_{1}+\mu_{\mathrm{r} 1}\right)\left(\mathrm{S}_{\mathrm{i}}+\lambda_{1}+\mathrm{a}_{2}+\mu_{\mathrm{r} 2}\right)+\lambda_{2}\left(\mathrm{a}_{2} \mathrm{X}\right. \\
& \left(\mathrm{S}_{\mathrm{i}}+\mu_{\mathrm{e} 2}\right)\left(\mathrm{S}_{\mathrm{i}}+\lambda_{1}+\mu_{\mathrm{e} 2}\right)\left(\mathrm{S}_{\mathrm{i}}+\lambda_{1}+\mathrm{a}_{2}+\mu_{\mathrm{r} 2}\right)+\lambda_{1} \mathrm{a}_{1}\left(\mathrm{~S}_{\mathrm{i}}+\mu_{\mathrm{e} 1}\right) \\
& \left(\mathrm{S}_{\mathrm{i}}+\lambda_{1}+\mu_{\mathrm{e} 1}\right)\left(\mathrm{S}_{\mathrm{i}}+\lambda_{1}+\mathrm{a}_{1}+\mu_{\mathrm{r} 1}\right)\left(\mathrm{a}_{2} \mu_{\mathrm{e} 2}\left(\mathrm{~S}_{\mathrm{i}}+\mathrm{a}_{2}+\mu_{\mathrm{r} 2}\right)+\left(\mathrm{S}_{\mathrm{i}}+\right.\right. \\
& \left.\left.\left.\lambda_{1}+\mu_{\mathrm{e} 2}\right)\left(\mathrm{a}_{2} \mu_{\mathrm{r} 2}+\mu_{\mathrm{r} 2}\left(\mathrm{~S}_{\mathrm{i}}+\mu_{\mathrm{e} 2}\right)\right)\right)\right)
\end{aligned}
$$

Where:

$$
\begin{aligned}
\mathrm{X}= & \left(( \mathrm { S } _ { \mathrm { i } } + \mathrm { a } _ { 1 } + \mu _ { \mathrm { r } 1 } ) \left(\left(\mathrm{S}_{\mathrm{i}}+\mu_{\mathrm{e} 1}\right)\left(\mathrm{S}_{\mathrm{i}}++\lambda_{1}+\mu_{\mathrm{e} 1}\right)\left(\mathrm{S}_{\mathrm{i}}+\lambda_{1}+\mathrm{a}_{1}+\mu_{\mathrm{r} 1}\right)\right.\right. \\
& \left.\left.-\mathrm{a}_{1} \lambda_{1} \mu_{\mathrm{e} 1}\right)-\lambda_{1}\left(\mathrm{~S}_{\mathrm{i}}+\lambda_{1}+\mu_{\mathrm{e} 1}\right)\left(\mathrm{a}_{1} \mu_{\mathrm{e} 1}+\mu_{\mathrm{r} 1}\left(\mathrm{~S}_{\mathrm{i}}+\mu_{\mathrm{e} 1}\right)\right)\right) \\
\mathrm{Y}= & \left(\mathrm{S}_{\mathrm{i}}+\mu_{\mathrm{e} 1}\right)\left(\mathrm{S}_{\mathrm{i}}+\mathrm{a}_{1}+\mu_{\mathrm{r} 1}\right)+\lambda_{1}\left(2 \mathrm{~S}_{\mathrm{i}}+\lambda_{1}+\mathrm{a}_{1}+\mu_{\mathrm{r} 1}+\mu_{\mathrm{e} 1}\right)
\end{aligned}
$$

Profit: The expected total profit per unit time incurred to the system in the steady state is given by:

$$
\mathrm{P}(\mathrm{t})=\mathrm{C}_{1} \mathrm{~A}_{0}-\mathrm{C}_{2} \mathrm{~B}_{0}-\mathrm{C}_{3} \mathrm{~T}_{0}-\mathrm{C}_{4} \mathrm{~V}_{0}
$$

\section{Graphical representation:}

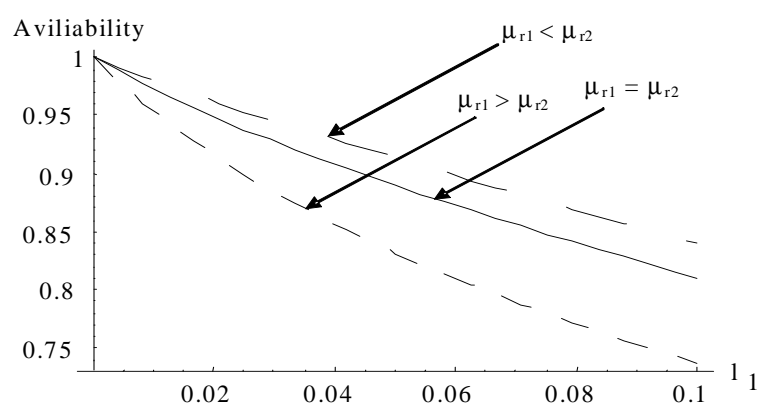

Fig. 1: Relation between availability and $\lambda_{1}$ with various values of $\mu_{\mathrm{r} 1}, \mu_{\mathrm{r} 2}$ 


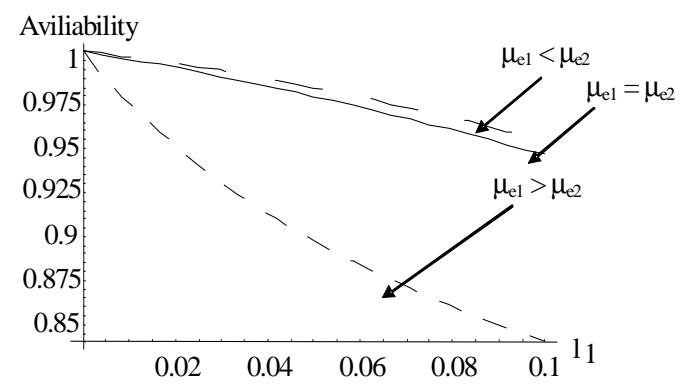

Fig. 2: Relation between availability and $\lambda_{1}$ with various values of $\mu_{\mathrm{e} 1}, \mu_{\mathrm{e} 2}$

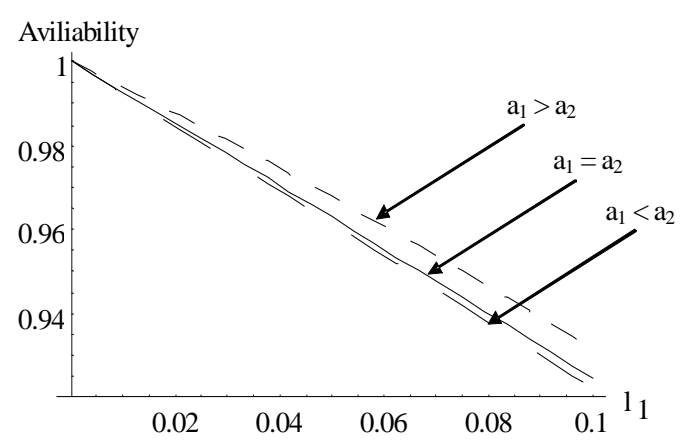

Fig. 3: Relation between availability and $\lambda_{1}$ with various values of $\mathrm{a}_{1}, \mathrm{a}_{2}$

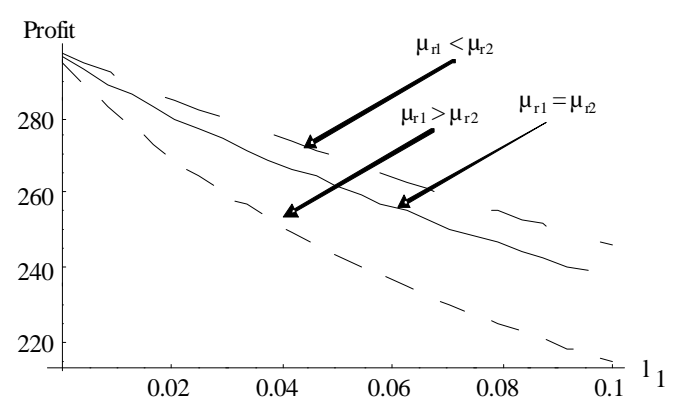

Fig. 4: Relation between profit and $\lambda_{1}$ with various values of $\mu_{\mathrm{r} 1}, \mu_{\mathrm{r} 2}$

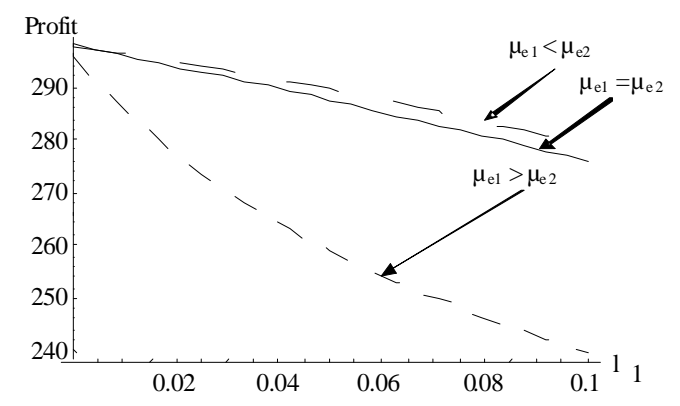

Fig. 5: Relation between profit and $\lambda_{1}$ with various values of $\mu_{\mathrm{e} 1}, \mu_{\mathrm{e} 2}$

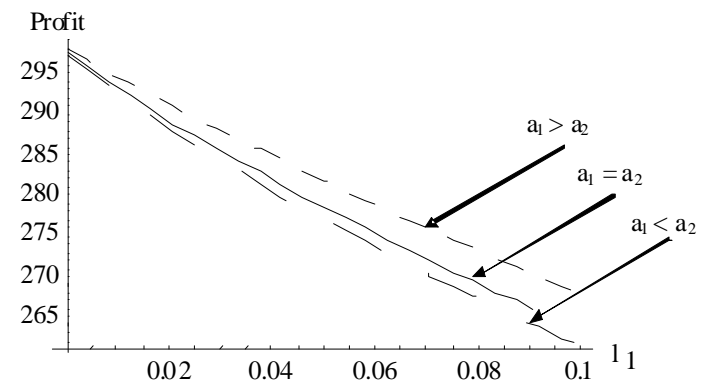

Fig. 6: Relation between profit and $\lambda_{1}$ with various values of $\mathrm{a}_{1}, \mathrm{a}_{2}$

\section{CONCLUSION}

By comparing the characteristic, availability and profit with respect to $\lambda_{1}$ for the system at fixed value of $\lambda_{2}$ and various values of $\mu_{\mathrm{r} 1}, \mu_{\mathrm{r} 2}, \mu_{\mathrm{e} 1}, \mu_{\mathrm{e} 2}, \mathrm{a}_{1}, \mathrm{a}_{2}$ the availability and profit of the system decrease with respect to the increase of $\lambda_{1}$.

We conclude that the availability and profit are improved when:

- $\mu_{\mathrm{r} 1}<\mu_{\mathrm{r} 2}$ than when $\mu_{\mathrm{r} 1}>\mu_{\mathrm{r} 2}$ as in Fig. 1 and 4

- $\mu_{\mathrm{e} 1}<\mu_{\mathrm{e} 2}$ than when $\mu_{\mathrm{e} 1}>\mu_{\mathrm{e} 2}$ as in Fig. 2 and 5

- $\mathrm{a}_{2}<\mathrm{a}_{1}$ than when $\mathrm{a}_{2}>\mathrm{a}_{1}$ as in Fig. 3 and 6

\section{REFERENCE}

1. Mahmoud, M.A.W. and M.A. Esmail, 1996. Probabilistic analysis of two-unit warm standby system subject to hardware and human error failures. Microelect. Reliabil., 36: 1565-1568. DOI: 10.1016/0026-2714(95)00181-6

2. Mokaddis, G.S. and M.L. Tawfek, 1996. Some characteristics of a two-dissimilar-unit cold standby redundant system with three modes. Microelect. Reliabil., 36: 497-503. http://cat.inist.fr/?aModele $=$ afficheN\&cpsidt $=3025970$

3. Wang, K.H., W.L. Dong and K.E. Jyh-Bin, 2006. Comparison of reliability and the availability between four systems with warm standby components and standby switching failures. Applied Math. Comput., 183: 1310-1322. http://cat.inist.fr/?aModele $=$ afficheN\&cpsidt $=18442281$

4. Sridharan, V. and P. Mohanavadivu, 1998. Stochastic behavior of a two unit cold standby system with two type of repairman and patience time. Math. Comput. Model., 28: 63-71. DOI: 10.1016/S0895-7177(98)00145-9 